\title{
A Secure Password Manager
}

\author{
Chaitanya Rahalkar \\ Savitribai Phule Pune University \\ H-404, Ruturang Society \\ Aranyeshwar Road, Pune
}

\author{
Dhaval Gujar \\ Savitribai Phule Pune University \\ 'Rajas Garden' Aparments \\ Model Colony, Pune
}

\begin{abstract}
Internet has grown exponentially over the past decade, and as a consequence, the amount of data generated is increasing day by day. Online services are growing and to keep online services personalised and organised, online accounts are being created by users. Over the past few years, incidents of data breaches have surfaced over the Internet, and there are some which are not even public knowledge. [8] Account passwords and personal information leaked from these data breaches are then misused or sold on the Internet. Cracking hashed passwords is not too difficult if the passwords among commonly used ones. [14] A Google / Harris Poll conducted in February 2019 concluded that 52\% people use the same password for multiple accounts. [6] Hence, even if one of them is compromised, all of their accounts are consequently compromised. To solve this problem, password managers were introduced. A password manager uses a master password that is the key to an encrypted vault. This vault contains critical data and passwords to various accounts. [10] It also generates secure passwords that ensure the security of one's account. The advantage that these password managers hold is that the user is required to remember just a single master password, instead of multiple passwords for different accounts. A single password can decrypt the encrypted vault allowing the user to access the password required for a particular account. They typically operate in either an offline or an online manner. Both require the use of a master password to unlock the rest of the passwords. [11] Both the approaches suffer from their own set of problems. The offline version requires that the file containing the encrypted passwords be transported everywhere and syncing the same file across many devices requires additional effort from the user, and if the file is lost, so are the passwords. The online version solves the sync and loss of file problem but an active Internet connection requirement is added, alongside the possibility of a security breach. Also, confidential and private data is stored on remote locations, which may produce a feeling of mistrust, if the underlying architectural details of the algorithms used and security of servers is kept hidden from the users. Data breaches may even occur on these servers. Thus, we propose an offline password manager, that does not store passwords anywhere. These passwords are not even stored on the device of the user, but are generated on-the-fly using the algorithm, by providing the master password.
\end{abstract}

\section{General Terms}

Password Security, Security, Cryptography

\section{Keywords}

Password Manager, Key Derviation Functions, Hash Functions, Security, Master Password

\section{INTRODUCTION}

The dependency on online services is increasing day by day, and thus large amount of confidential data like credit card numbers, bank account details, account passwords are being stored on servers, always under the threat of getting stolen in a data breach incident. People tend to keep easy to remember passwords, and this is what hackers take advantage of. Passwords containing names, phone numbers etc. are easily guessable or can be cracked easily with a wordlist. A secure password must contain arbitrary numbers, characters and special characters and must be at least 8 characters long. Brute forcing these passwords would seem infeasible if they are long enough, containing arbitrary characters and numbers. Keeping secure, non-guessable passwords should be the highest priority when creating an online account, keeping in mind the consequences of not doing so. Using arbitrary passwords is the safest way to secure an online account. The state-less master password algorithm was initially proposed by Maarten Billemont [3], and this algorithm is a modified and a more secure version of his proposal with support for encrypted file sharing. The master password manager can be implemented as a Web App, Phone App or Desktop application using standard cryptographic libraries, thereby making it available on all platforms

\section{RELATED WORK}

Password managers are not a new concept. They have been in existence for a decade now, but have almost always been stateful. Companies like 1Password [5], LastPass [1] and open source password managers like KeePass [2] have been used predominantly. These password managers usually come as a bundle of three features -

(1) A random and secure password generator (which generates secure random strings to be used as passwords)

(2) An encrypted storage vault to store all the passwords

(3) A two-factor authentication code generator.

Some password managers implement a local database to store all the passwords while some use remote encrypted online stores. These password managers are accessible through web applications or mobile applications. Apart from these, hardware devices are also being used as password managers. [7] On inserting the device to 
a computer via the USB port, the password is entered on behalf of the user. All the passwords are stored on the hardware device. These hardware device manufacturers claim to have military grade security on these hardware password managers. [4]

\section{PROBLEMS}

Using password managers that use a single master password to encrypt all your account password and information require a central authority that stores the encrypted data on a server. Retrieving these passwords from the server requires an active Internet connection. To tackle this, the password managers tend to store the encrypted vault on the user's personal device. If the device is stolen / lost, or if the master password is not strong enough, all the data may be compromised. [9] Storing private data on central servers may often produce a feeling of mistrust since there is always a chance of data getting breached. Even though hardware password managers are secure, it involves carrying the hardware device everywhere, and if the device is lost, since no online backups are kept, all the password data is lost. Some password managers are local-storage based, but use a web interface to interact with the user. These password managers are vulnerable to JavaScript code injection. [12]

A small flaw in the algorithm design may break the complete system. Also, one has to ensure that the data from the server is synced with multiple instances of the app on various platforms like mobile, computers, smart watches etc. If they are not in sync, a newly added password to the vault, may not be accessible from a different or new device. A personal device on which the password manager app is stored is always needed in order to access the passwords.

\section{PROPOSAL}

The master password generator algorithm is an algorithm that doesn't store passwords anywhere. The setting up stage starts with the user selecting a master password. Then, the user enters the website for which he / she wants a password. Then the user selects the type of password - numeric, alphanumeric, character only, or a passphrase. The algorithm generates a new, secure and unique password for the website. The user then sets this generated password as the password for the website. This algorithm ensures that every time you enter the master password and the website name correctly, it will produce the same password as generated initially. Thus, passwords are generated on-the-fly. The only inconvenience involved in this method is changing the existing insecure website passwords to these newly generated passwords, which is to be done just once. After the initial setup phase, the algorithm will always generate the desired password, provided that the master password, website name and password type are the same. This algorithm does not restrict itself to generating arbitrary passwords, it can also generate pin numbers, pass-phrases (A pass-phrase is a set of arbitrary but meaningful words used together as a password. They are easy to remember due to usage of common words) uniquely produced by the master password. The major advantage of this password manager is that there is no way of breaching any sort of data. Also there is no way for the attacker to know whether his/her guess of the master password is correct or not, since the master password algorithm will always have a unique set of passwords associated with the master password.

The algorithm can be implemented as a web, mobile or a standalone desktop application, with the same algorithm implemented on all platforms. This will ensure that a password generated on a web app

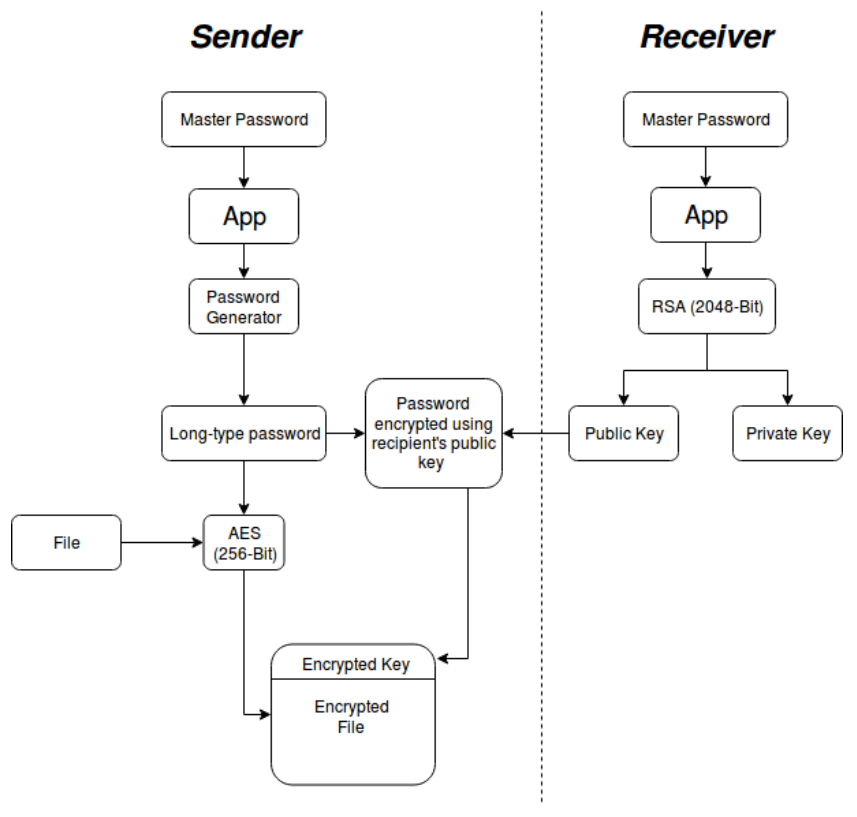

Fig. 1. File Encryption Approach Similar to PGP

can be regenerated on-the-fly on the mobile or desktop application without the need of any synchronisation mechanism.

An additional feature implemented with this algorithm is that a key generated from the master password can be used to encrypt files. The file encryption scheme follows a similar approach to PGP. The file is encrypted using a symmetric key with AES-256 bit encryption. This is explained in Fig.1.

This symmetric key is generated from the master password and then encrypted with the RSA 2048-bit public key of the recipient. The decryption is exactly the reverse process, where the RSA decryption is done using the 2048-bit private key of the recipient in order to recover the symmetric key which is then used to decrypt the AES-256 bit encrypted file.

\section{ALGORITHM}

Deriving a password for a website from the master password involves three stages:

(1) Generation of a secret key from the master password. The secret key generation process uses a key derivation function. A key derivation function (KDF) derives one or more secret keys from a secret value such as a master key, a password, or a passphrase using a pseudorandom function. KDFs are deliberately made slow to compute so as to make brute-force attacks or dictionary attacks more strenuous. The proposed algorithm uses Argon2 as the KDF. Specifically, it uses Argon2d (one of the versions of Argon). The main advantage of using Argon $2 \mathrm{~d}$, is that it maximises resistance to GPU cracking attacks. Till date, no public cryptanalysis methods have been published which are applicable to Argon2d.

The function prototype can be defined as follows: 


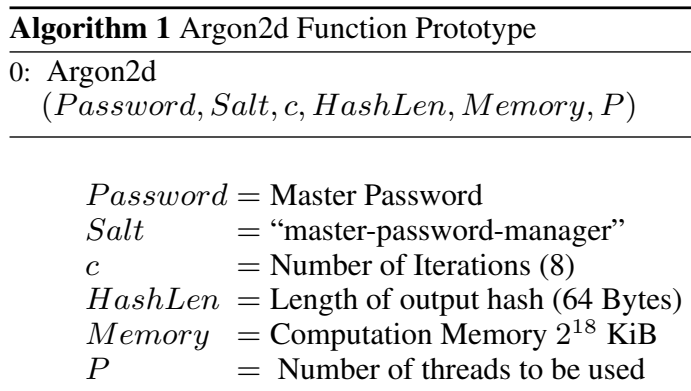

The intensity of computation can be controlled with the cost factor (c) (Number of iterations) in the algorithm. Greater the number of iterations, more is the compute time required for deriving the key. Such KDF algorithms are widely used in cryptocurrencies as Proof-of-Work algorithms. [13] Hash cracking speeds can be intensified by using specially designed hardware called ASICs (Application Specific Integrated Circuits). However, Argon2d is resistant to such attacks, since it is a memory hard function that maximizes the ASIC implementation costs. The output of this function is a 64-bit key called as the derived key.

(2) A key unique to every site is generated from the previously generated derived key. The second stage uses HMAC (Hashbased Message Authentication Codes) with the hashing algorithm being SHA-256, to generate a unique site-key. This site key is 256-bits or 32-bytes in output. The message is a string concatenation of the length of site-name string, the site-name and a counter. The counter is a third parameter introduced to completely minimize the chances of brute-forcing the password. The user decides on a counter value which can be any integer value from 1 to $2^{64}$. The counter value is set to 1 by default if the user doesn't provide one. Thus, from an attacker's perspective, getting to the correct password would involve guessing the master password, the website name and the counter value, all of them correctly.

$$
\text { Site }- \text { Key }=\text { HMAC-SHA-256 (message, key) }
$$

where:

$$
\begin{aligned}
\text { message } & =\text { length }(\text { sitename }) \cdot \text { sitename } \cdot \text { counter } \\
\text { key } & =\text { Secret }- \text { Key }
\end{aligned}
$$

(3) A template is selected depending upon the type of password to be generated. The user can choose from the following password types:

Maximum security password - This password is a combination of special characters, numbers and alphabets. It is 21 characters in length.

Long Password - It may contain letters, numbers and special characters having lesser frequency as compared to the maximum security type. It is 15 characters in length.

Medium Password - It may contain letters, numbers and only a single special character. It is 9 characters in length. Basic Password - It consists of only letters and numbers. It is 9 characters in length.

Short Password - It is a very small password containing of just 4 characters out of which one is a number. These passwords can be used for phone pins or application passwords on phones.

Pin Password - It is a 4 digit numeric pin. This pin can be

\begin{tabular}{|c|c|c|}
\hline Sr No. & Password Type & Template \\
\hline 1 & Maximum Length & 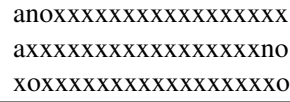 \\
\hline 2 & Long & 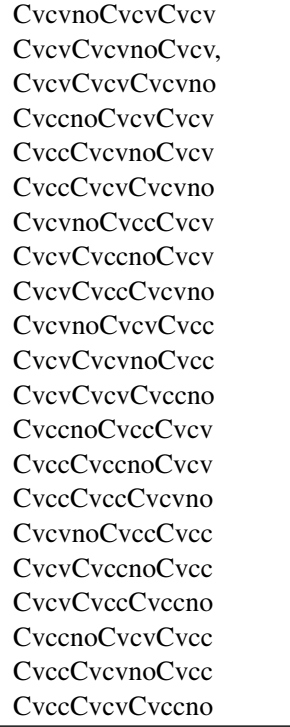 \\
\hline 3 & Medium & $\begin{array}{l}\text { CvenoCve } \\
\text { CveCveno }\end{array}$ \\
\hline 4 & Basic & $\begin{array}{l}\text { aaanaaan } \\
\text { aannaaan } \\
\text { aaannaaa }\end{array}$ \\
\hline 5 & Short & Cven \\
\hline 6 & Pin & nnnn \\
\hline 7 & Short (Characters Only) & crecvevev \\
\hline
\end{tabular}
used for mobile passwords, ATM pins etc.
Table 1. Password Templates

Pass-phrase - A pass phrase consists of valid dictionary words, instead of arbitrary characters. A pass-phrase is easy to remember due to the presence of actual words. These words are selected by the algorithm from a list of 10000 English words to form a phrase. The phrase may or may not have any meaning. It is a combination of pronounceable, valid English words.

Symmetric Key - This is a special type of password derived from the master password which is used to encrypt files with AES-256 bit encryption.

The master password algorithm uses a template scheme to generate passwords.The different templates used are shown in Table 1.

\section{where}

$C=$ BCDFGHJKLMNPQRSTVWXYZ (Capital cased consonants)

$v=$ aeiou (Small cased vowels)

$V=$ AEIOU (Capital cased vowels)

$c=$ bcdfghjklmnpqrstvwxyz (Small cased consonants)

$n=0123456789$ (Numbers)

$o=@ \& \% ?,=[]:-+\$ \# !^{\prime} ;() / .($ Special characters $)$

$x=($ Special characters + Numbers + Small and capital letters $)$

$a=$ (Small and capital letters)

The following pseudo-code explains the password generation procedure - 


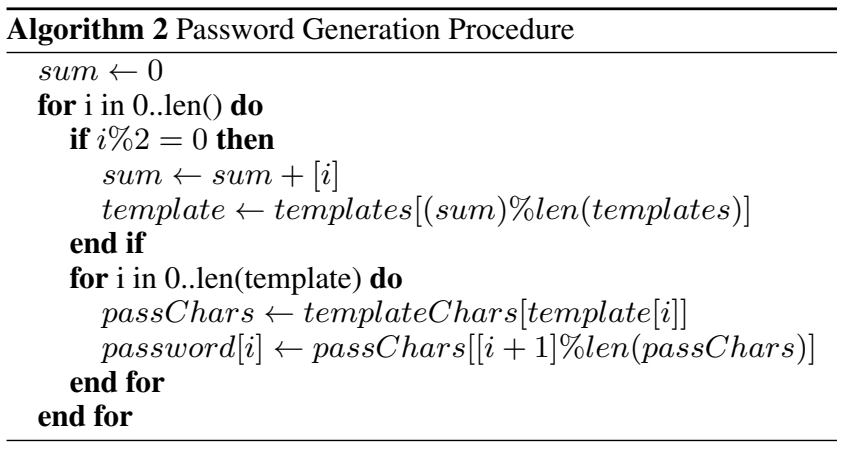

For generating a passphrase a template is not used. Words are selected from a dictionary of pronounceable English words. The pseudo-code is different.

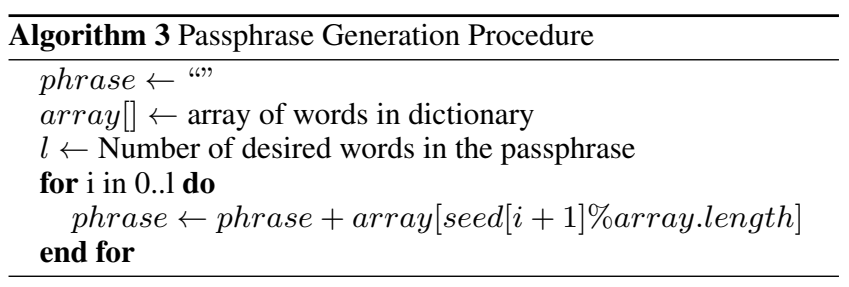

Brief explanation of the pseudo-code:

i. The output string that is generated is in hexadecimal. Hexadecimal sum of even bits of the site-key is calculated.

ii. A template is chosen from the list of templates for the specified password type, by performing simple modulus operation with the total number of templates available for the password type. The modular property will ensure that a template within the specified list is selected.

iii. Now on the similar lines, depending upon the type of character observed in the template selected, a character from that list is selected by performing modulus operation with the total number of characters available in the type.

iv. A site password is generated as per the selected type.

\section{CONCLUSION}

Our paper throws light on the problems faced by traditional password managers and highlights why it is necessary to move on to state-less password managers and presents an algorithm for the same.

Our approach uses state-of-the-art cryptography techniques to deliver a safe, secure, yet uncompromising password management application that is:
i. Fast
ii. Generates secure passwords of varied types
iii. State-less (No database of any kind)
iv. Portable
v. Does not need any synchronisation
vi. Can be used by multiple people on the same device

\section{FUTURE SCOPE}

The algorithm can be deployed as a complete password manager application, running cross-platform. An extension to this can be to creation of a browser plugin / extension that can be used to auto-fill passwords on websites. [15]

The password generation algorithm is not restricted to a particular programming language. The accessibility of the password manager is crucial and hence it should be implementable on a variety of platforms which includes websites, phone applications and desktop applications.

It may also be possible to create a stand-alone hardware device that will have a biometric sensor, USB HID (Human Interface Device) capability and buttons for site selection. This device can be plugged into any host device that accepts USB keyboards, and the generated passwords can then be entered, driver-lessly. However, for such a hardware to exist, algorithms that can produce a hash based on fingerprint minutiae [16] must be studied and carefully applied. Such a hardware device will be cost effective to construct, would work for any person (no storage and no vendor lock-in) and on any device which accepts USB HID keyboard input.

If device manufacturers deem fit, all upcoming devices can implement this functionality by default, virtually eliminating the need to ever remember passwords or use weak ones.

\section{REFERENCES}

[1] \#1 Password Manager \& Vault App, Enterprise SSO \& MFA | LastPass. https://www.lastpass.com/

[2] keypass.info.https://keepass.info/

[3] Master Password: Home. www.masterpasswordapp.com/

[4] OnlyKey Hardware Password Manager | One PIN to remember.https://onlykey.io/.

[5] Password Manager for Families, Businesses, Teams. http: //1password.com/

[6] Password Practices Still Poor, Google Says SecurityWeek.Com.https://www.securityweek.com/passwordpractices-still-poor-google-says

[7] Portable password manager. https:// patents.google.com/patent/US20040193925A1/en March 2004.

[8] Information is Beautiful. World's Biggest Data Breaches \& Hacks. https://informationisbeautiful.net/ visualizations/worlds-biggest-data-breacheshacks/

[9] Paolo Gasti and Kasper B. Rasmussen. On the Security of Password Manager Database Formats. In Sara Foresti, Moti Yung, and Fabio Martinelli, editors, Computer Security - ESORICS 2012, Lecture Notes in Computer Science, pages 770787. Springer Berlin Heidelberg, 2012.

[10] Alexa Huth, Michael Orlando, and Linda Pesante. Password security, protection, and management. 2012.

[11] Ambarish Karole, Nitesh Saxena, and Nicolas Christin. A Comparative Usability Evaluation of Traditional Password Managers. In Kyung-Hyune Rhee and DaeHun Nyang, editors, Information Security and Cryptology - ICISC 2010, Lecture Notes in Computer Science, pages 233-251. Springer Berlin Heidelberg, 2011. 
[12] Zhiwei Li, Warren He, Devdatta Akhawe, and Dawn Song. The Emperor's New Password Manager: Security Analysis of Web-based Password Managers. pages 465-479, 2014.

[13] Colin Percival and Simon Josefsson. The scrypt passwordbased key derivation function. 2016.

[14] Steve Ragan. Thousands of gamers' passwords easily cracked in 3 minutes. https://www.csoonline.com/article/ 3025628/ignore-the-worlds-worst-passwordslook-at-how-theyre-created-instead.html. January 2016.

[15] David Silver, Suman Jana, Dan Boneh, Eric Chen, and Collin Jackson. Password Managers: Attacks and Defenses. pages 449-464, 2014.

[16] Sergey Tulyakov, Faisal Farooq, Praveer Mansukhani, and Venu Govindaraju. Symmetric hash functions for secure fingerprint biometric systems. Pattern Recognition Letters, 28(16):2427-2436, December 2007. 